\title{
Clearance of inflammatory cytokines in patients with septic acute kidney injury during renal replacement therapy using the EMiC2 filter (Clic-AKl study)
}

\author{
Nuttha Lumlertgul ${ }^{1,2,3^{*}}$, Anna Hall ${ }^{1,4}$, Luigi Camporota', Siobhan Crichton ${ }^{5}$ and Marlies Ostermann ${ }^{1}$ (i)
}

\begin{abstract}
Background: The EMiC2 membrane is a medium cut-off haemofilter (45 kiloDalton). Little is known regarding its efficacy in eliminating medium-sized cytokines in sepsis. This study aimed to explore the effects of continuous venovenous haemodialysis (CVVHD) using the EMiC2 filter on cytokine clearance.

Methods: This was a prospective observational study conducted in critically ill patients with sepsis and acute kidney injury requiring kidney replacement therapy. We measured concentrations of 12 cytokines [Interleukin (IL) IL-1 $\beta, \mathrm{IL}-1 \mathrm{a}$, IL-2, IL-4, IL-6, IL-8, IL-10, interferon (IFN)- $y$, tumour necrosis factor (TNF)- $a$, vascular endothelial growth factor, monocyte chemoattractant protein (MCP)-1, epidermal growth factor (EGF)] in plasma at baseline (T0) and pre- and postdialyzer at 1, 6, 24, and $48 \mathrm{~h}$ after CVVHD initiation and in the effluent fluid at corresponding time points. Outcomes were the effluent and adsorptive clearance rates, mass balances, and changes in serial serum concentrations.
\end{abstract}

Results: Twelve patients were included in the final analysis. All cytokines except EGF concentrations declined over $48 \mathrm{~h}(p<0.001)$. The effluent clearance rates were variable and ranged from negligible values for IL-2, IFN- $\gamma$, IL-1 a, IL-1 $\beta$, and EGF, to $19.0 \mathrm{ml} / \mathrm{min}$ for TNF-a. Negative or minimal adsorption was observed. The effluent and adsorptive clearance rates remained steady over time. The percentage of cytokine removal was low for most cytokines throughout the 48-h period.

Conclusion: EMiC2-CVVHD achieved modest removal of most cytokines and demonstrated small to no adsorptive capacity despite a decline in plasma cytokine concentrations. This suggests that changes in plasma cytokine concentrations may not be solely influenced by extracorporeal removal.

Trial registration: NCT03231748, registered on 27th July 2017.

Keywords: EMiC2 filter, Middle cut-off, High cut-off, Extracorporeal blood purification, Sepsis, Removal, Acute kidney injury, CRRT, Kidney replacement therapy

\footnotetext{
*Correspondence: Nuttha.Lumlertgul@gstt.nhs.uk

1 Department of Critical Care, Guy's and St Thomas' Hospital, King's

College London, NHS Foundation Trust, 249 Westminster Bridge Road, London SE1 7EH, UK

Full list of author information is available at the end of the article
}

\begin{abstract}
Introduction
Sepsis is a life-threatening condition in which a dysregulated release of pro- and anti-inflammatory cytokines can lead to multiple organ failure and increased mortality [1]. Management of sepsis is supportive [2].

Critically ill patients with sepsis-associated acute kidney injury (AKI) requiring kidney replacement therapy
\end{abstract} original author(s) and the source, provide a link to the Creative Commons licence, and indicate if changes were made. The images or other third party material in this article are included in the article's Creative Commons licence, unless indicated otherwise in a credit line to the material. If material is not included in the article's Creative Commons licence and your intended use is not permitted by statutory regulation or exceeds the permitted use, you will need to obtain permission directly from the copyright holder. To view a copy of this licence, visit http://creativecommons.org/licenses/by/4.0/. The Creative Commons Public Domain Dedication waiver (http://creativeco mmons.org/publicdomain/zero/1.0/) applies to the data made available in this article, unless otherwise stated in a credit line to the data. 
(KRT) are at particularly increased risk of death [3]. However, KRT may offer the opportunity to provide extracorporeal blood purification therapy (BPT). With regards to the potential benefit of $\mathrm{BPT}$, there are several hypotheses. The 'peak concentration hypothesis' proposes that during BPT excessive pro- or anti-inflammatory mediators are removed and plasma concentrations decrease to levels below a toxic threshold [4, 4]. The 'cytokinetic theory' proposes that cytokine removal creates a decreased cytokine gradient between tissues and the bloodstream and promotes leukocyte chemotaxis towards the infected tissues [6]. The 'cytotoxic threshold immune modulation theory' relates to the removal of cytokines from the cytokine-concentrated interstitium and tissues into the circulation [7]. Finally, a 'cellular theory' refers to removing leucocytes directly or through immune cell reprogramming $[8,9]$. The proposed techniques include high-volume haemofiltration (HVHF), use of high cut-off (HCO) membrane and adsorption techniques, or plasmapheresis [10]. HCO haemofiltration with a cut-off up to $60 \mathrm{kDa}$ has been shown to achieve higher cytokine clearance than conventional membranes (15-30 kDa), but the effects on clinical outcomes such as haemodynamic improvement, severity scores, and survival are inconclusive [11-17]. Besides, the concomitant loss of albumin, proteins, micronutrients, and antibiotics is a concern $[18,19]$.

The EMiC2 filter (Fresenius, Bad Homburg, Germany) is a polysulfone (PS)-based membrane with a cut-off of $45 \mathrm{kDa}$. Case reports showed reduction in serum $\mathrm{k}$-free light chains and myoglobin levels with the $\mathrm{EMiC} 2$ filter, but actual removal by the filter was not investigated [2023]. Other studies reported higher removal of kappa light chains (23 kDa), $\beta 2$-microglobulin (17 kDa), myoglobin $(17 \mathrm{kDa}), \mathrm{IL}-6$, and IL-8 in patients receiving treatment with $\mathrm{EMiC2}$ filters compared with standard high-flux membranes [24-27]. EMiC2-continuous veno-venous haemodialysis (CVVHD) was well-tolerated, and albumin loss was limited [28]. However, in-vitro data showed no adsorptive capacity of the $\mathrm{EMiC} 2$ filter compared with other membranes [29].

The exact role of the EMiC2 filter in the treatment of sepsis-associated AKI is unknown. Furthermore, the causal link between reduction in plasma cytokine concentration and extracorporeal cytokine removal has not been demonstrated $[26,28]$. Before proceeding to a trial comparing the $\mathrm{EMiC2}$ filter with other commercially available filters to manage sepsis-associated AKI, it is important to determine the characteristics and the actual magnitude of cytokine removal in vivo. In this pilot study, we aimed to measure the clearance of middle molecular weight cytokines using the $\mathrm{EMiC} 2$ filter in patients receiving CVVHD.

\section{Material and methods}

This study was a prospective observational study in a 62-bed mixed tertiary-care intensive care unit (ICU) between June and September 2017. The study was approved by the Research Ethics Committee $(16 / \mathrm{LO} / 0313)$, registered on clinicaltrials.gov (NCT03231748) and conducted in accordance with the Declaration of Helsinki 2013. Written informed consent was obtained from all patients or their legal representatives.

\section{Subjects}

Eligible patients were critically ill adult patients with acute kidney injury and sepsis in whom a decision had been made by the clinical team to start citrate-based CVVHD. Exclusion criteria were lack of consent, preexisting dialysis-dependent kidney failure, life expectancy $<24 \mathrm{~h}$, haemoglobin $<7 \mathrm{~g} / \mathrm{dL}$, and need for extracorporeal membrane oxygenation (ECMO).

\section{Kidney replacement therapy (KRT) setting}

CVVHD was performed with the dialysis machine multifiltrate using the medium cut-off dialyzer $\mathrm{EMiC} 2$ and a bicarbonate-buffered dialysate (Fresenius Medical Care, Bad Homburg, Germany) at $25-30 \mathrm{ml} / \mathrm{kg} / \mathrm{h}$ [30]. Regional citrate anticoagulation was used in all patients.

\section{Cytokine measurement}

The concentrations of interleukin (IL)-2, IL-4, IL-6, IL-8, IL-10, vascular endothelial growth factor (VEGF), interferon gamma (IFN- $\gamma$ ), tumour necrosis factor alpha (TNF- $\alpha$ ), IL-1alpha (IL-1 $\alpha$ ), IL-1beta (IL-1 $\beta)$, monocyte chemoattractant protein-1 (MCP-1), epidermal growth factor (EGF) were measured before initiation of CVVHD (T0) and pre- and post-dialyzer during CVVHD at 1, 6, 24, and $48 \mathrm{~h}$ (T1, T6, T24, and T48, respectively) (Additional file 1). These molecules were also measured in the effluent at the same time points (T1, T6, T24, and T48). If CVVHD had to be temporarily discontinued, sampling was performed 1-2 $\mathrm{h}$ after CVVHD was re-started using the same circuit. In case, a circuit change was necessary within the first $24 \mathrm{~h}$ of the study, sampling was recommenced de novo with the new filter. If a further filter change was necessary within the first $24 \mathrm{~h}$, the patient was withdrawn from the study.

\section{Laboratory analyses}

Blood and effluent samples were centrifuged at $3000 \mathrm{rpm}$ for $15 \mathrm{~min}$ and stored in $\mathrm{a}-80{ }^{\circ} \mathrm{C}$ freezer until batch analysis at the end of the study. The cytokine concentrations were determined by electro-chemiluminescent immunoassay (ECLIA) method using an Evidence 
Investigator Bioship system (Randox Laboratories Limited, the United Kingdom).

\section{Outcomes}

The primary outcome of interest was cytokine clearance during EMiC2-based CVVHD. The secondary endpoints were adsorption by the EMiC2 filter, changes in cytokine concentrations in plasma, and reduction ratios of all cytokines over $48 \mathrm{~h}$.

\section{Clearances}

Effluent clearance $\left(\mathrm{Cl}_{\mathrm{eff}}\right)$ at each sampling time point was estimated using the following equation [31]:

$$
\mathrm{Cl}_{\mathrm{eff}}\left(\frac{\mathrm{ml}}{\min }\right)=\frac{Q_{\mathrm{df}}}{60} \times \frac{\text { Ceffluent }}{(\text { Cpredialyzer }+ \text { Cpostdialyzer }) / 2}
$$

where $Q_{\mathrm{df}}$ represents dialysate flow rate $(\mathrm{ml} / \mathrm{h})$.

Adsorptive clearance $\left(K_{\mathrm{ad}}\right)$ was calculated as:

$$
\operatorname{Kad}\left(\frac{\mathrm{ml}}{\min }\right)=\frac{M_{\mathrm{ad}}}{\text { Cpredialyzer }}
$$

$M_{\text {ad }}$ represents mass removal rate by membrane adsorption (pg/min) (see below):

The average hourly effluent clearance $\left(\mathrm{Cl}_{\text {total }}\right)$ during the study period was calculated using the following formula:

$$
\begin{aligned}
& \mathrm{Cl}_{\text {total }}\left(\frac{\mathrm{ml}}{47}\right)=\frac{\mathrm{Cl} 1 \mathrm{~h}+\mathrm{Cl} 6 \mathrm{~h}}{2} \times 5 \times 60 \\
& \quad+\frac{\mathrm{Cl} 6 \mathrm{~h}+\mathrm{Cl} 24 \mathrm{~h}}{2} \times 18 \times 60+\frac{\mathrm{Cl} 24 \mathrm{~h}+\mathrm{Cl} 48 \mathrm{~h}}{2} \times 24 \times 60
\end{aligned}
$$

$$
\begin{aligned}
& M_{\text {postdialyzer }}=Q_{\mathrm{o}} \times C_{\text {postdialyzer }} ; Q_{\mathrm{o}}=Q_{\mathrm{i}}-Q_{\mathrm{uf}} \\
& M_{\text {total }}=M_{\text {predialyzer }}-M_{\text {postdialyzer }} \\
& M_{\mathrm{df}}=Q_{\mathrm{df}} \times C_{\mathrm{eff}} \\
& \mathrm{M}_{\mathrm{ad}}=\mathrm{M}_{\mathrm{total}}-\mathrm{M}_{\mathrm{df}}
\end{aligned}
$$

where $Q_{\mathrm{i}}$ is inlet plasma flow rate $(\mathrm{ml} / \mathrm{min}) ; Q_{\mathrm{b}}$ is blood flow rate $(\mathrm{ml} / \mathrm{min})$; Hct is haematocrit at sampling time; $Q_{\mathrm{o}}$ is outlet plasma flow rate $(\mathrm{ml} / \mathrm{min}) ; Q_{u f}$ is ultrafiltration rate $(\mathrm{ml} / \mathrm{min}) ; Q_{\mathrm{df}}$ is dialysate flow rate $(\mathrm{ml} / \mathrm{min})$; $M_{\text {predialyzer }}$ is inlet mass rate ( $\left.\mathrm{pg} / \mathrm{min}\right) ; M_{\text {postdialyzer }}$ is outlet mass rate $(\mathrm{pg} / \mathrm{min}) ; M_{\text {total }}$ is total mass removal rate $(\mathrm{pg} /$ $\min ) ; M_{\mathrm{df}}$ is mass removal rate by CVVHD (pg/min); $M_{\mathrm{ad}}$ is mass removal rate by membrane adsorption $(\mathrm{pg} / \mathrm{min})$.

We only included subjects with detectable pre-dialyzer concentrations when analysing the effluent and adsorptive clearance rates and mass balances.

\section{Reduction ratios}

The reduction ratio (RR) of plasma cytokine concentrations at each time point was calculated as follows [24]:

$$
\mathrm{RR}=\frac{\text { Cpredialyzer timeX }- \text { Ctime0 }}{\text { Ctime } 0} \times 100
$$

The period between starting CVVHD and the 1-h time point was not included due to the necessary equilibration process. Average clearance per minute $\left(\mathrm{Cl}_{\text {mean }}\right)$ was determined from $\mathrm{Cl}_{\text {total }}$ as follows:

$$
\mathrm{Cl}_{\text {mean }}\left(\frac{\mathrm{ml}}{\min }\right)=\frac{\mathrm{Cl}_{\text {total }}}{47 \times 60}
$$

\section{Mass balance}

Mass balance equations describe the transport of molecules and account for material entering and leaving a system. They allow the estimation of contribution from adsorption and removal into the effluent. Mass balance of the cytokines at each time point was calculated as follows:

$$
M_{\text {predialyzer }}=Q_{\mathrm{i}} \times C_{\text {predialyzer }} ; Q_{\mathrm{i}}=Q_{\mathrm{b}} \times\left(1-\frac{\mathrm{Hct}}{100}\right)
$$

where $\mathrm{Ctime} 0=$ plasma concentrations of cytokines at baseline before CVVHD initiation.

\section{Statistical analyses}

The Kolmogorov-Smirnov test was performed to test for normal distribution of continuous variables. Normally distributed data were summarised as mean \pm standard deviation. Missing data were not imputed. Nonparametric variables were summarised as median with interquartile range. Changes in median levels over time were compared using generalised estimating equations (GEE). Spearman's correlation was performed to assess the correlation between plasma cytokine concentrations and clearance rates. Linear regression was performed to investigate the link between molecular weight and clearance. A $p$ value $<0.05$ was considered statistically significant. Data were analysed using Stata 16 (StataCorp, College Station, Texas). 


\section{Results}

\section{Patient characteristics}

Thirteen patients were recruited to the study, but one patient was excluded because KRT was not started for clinical reasons. Baseline characteristics, severity scores, clinical and laboratory data at KRT initiation of the remaining 12 patients are presented in Table 1 . The median dialysate volume was $2400 \mathrm{~mL}$ (IQR 2300-3000), and the median ultrafiltration rate was $40 \mathrm{~mL} / \mathrm{h}$ (IQR 0-190). Eight patients were discharged alive from the ICU.

\section{Cytokine plasma concentrations}

Median and interquartile range of pre-dialyzer plasma cytokine concentrations at baseline and pre-determined time points are displayed in Table 2. IL-2, EGF, IFN- $\gamma$, IL- $1 \beta$ were undetectable in $3,3,2$, and 1 patient, respectively, throughout the whole study period. The plasma concentrations of all cytokines except EGF significantly decreased over time $(p<0.001)$. Figure 1 demonstrates

\section{Table 1 Baseline characteristics of study patients before CVVHD initiation}

\begin{tabular}{|c|c|}
\hline Parameters & $n=12$ \\
\hline \multicolumn{2}{|l|}{ Baseline data } \\
\hline Age (median, IQR) & $57(47.8,75)$ \\
\hline Male (n) & 8 \\
\hline \multicolumn{2}{|l|}{ Source of sepsis (n) } \\
\hline Lung & 5 \\
\hline Abdomen & 5 \\
\hline Musculoskeletal system & 2 \\
\hline \multicolumn{2}{|l|}{ Comorbidities (n) } \\
\hline Diabetes & 3 \\
\hline Hypertension & 6 \\
\hline Chronic heart disease & 3 \\
\hline Chronic lung disease & 1 \\
\hline Cerebrovascular disease & 3 \\
\hline Weight (kg) & $72.5(70,87)$ \\
\hline Height $(\mathrm{cm})$ & $176(165,179)$ \\
\hline \multicolumn{2}{|l|}{ Parameters on day of enrolment } \\
\hline Mechanical ventilation (n, \%) & $9(75 \%)$ \\
\hline Vasopressor use $(\mathrm{n}, \%)$ & $9(75 \%)$ \\
\hline APACHE II (median, IQR) & $27(23,30)$ \\
\hline SOFA score (median, IQR) & $11(10,12)$ \\
\hline Urine output (mL/day) & $63(33,161)$ \\
\hline \multicolumn{2}{|l|}{ Laboratory data (median, IQR) } \\
\hline Haemoglobin (g/L) & $86(71,104)$ \\
\hline Urea (mmol/L) & $13.1(11.5,18)$ \\
\hline Creatinine $(\mu \mathrm{mol} / \mathrm{L})$ & $287(150,438)$ \\
\hline C-reactive protein (mg/L) & $127(82,228)$ \\
\hline
\end{tabular}

$I Q R$ interquartile range, APACHE Acute Physiologic and Chronic Health Evaluation II, SOFA Sequential Organ Failure Assessment the pre-filter cytokine concentrations at each time point relative to baseline levels (T0,100\%). At $48 \mathrm{~h}$, the pre-filter cytokine concentrations decreased to $38.98 \pm 18.89 \%$ for IFN- $\gamma$ and to $90.57 \pm 52.21 \%$ for IL-2, corresponding to reduction ratios of $-61.02 \%$ and $-9.43 \%$, respectively. (Additional file 2) In contrast, for EGF, the pre-filter cytokine concentrations at $48 \mathrm{~h}$ were $161.74 \pm 97.84 \%$ higher compared with baseline.

\section{Cytokine removal \\ Clearance rates}

The adsorption and effluent clearance rates at each time point are shown in Table 3 and Additional file 3 . The median time-weighted effluent clearance rates varied from $0 \mathrm{~mL} / \mathrm{min}$ in 5 cytokines (IL-2, IFN- $\gamma$, IL- $1 \alpha$, IL- $1 \beta$, and EGF) to $33.9 \mathrm{~mL} / \mathrm{min}$ (MCP-1), $55.4 \mathrm{~mL} / \mathrm{min}$ (IL4), and $63.8 \mathrm{~mL} / \mathrm{min}$ (IL-8). The effluent clearance rates were constant during the 48 -h period for most cytokines, except for IL-10 and TNF- $\alpha$ where clearance rates were higher at T48. The median time-weighted adsorption rates ranged from $-64.0 \mathrm{ml} / \mathrm{min}$ (IQR -91.2 to -43.4 ) for IL-4 to $9.8 \mathrm{ml} / \mathrm{min}$ (IQR -3.8 to 46.3 ) for IL-2. Negative values for adsorption were observed in all cytokines at some time points. There were no significant changes in adsorption rates over time. Correlations between serum concentrations and adsorption and effluent clearance are shown in Additional file 4.

\section{Mass balance}

The total mass transfer $\left(M_{\text {total }}\right)$ and mass balance via adsorption $\left(M_{\mathrm{ad}}\right)$ and CVVHD $\left(M_{\mathrm{df}}\right)$ are demonstrated in Additional file 5. Total mass transfer and the contributions from $M_{\mathrm{df}}$ and $M_{\mathrm{ad}}$ were calculated in percentage of $M_{\text {predialyzer }}$ and shown in Fig. 2. There was marked heterogeneity in the proportion of haemofilter clearance and adsorption for all cytokines over time. CVVHD $\left(\% M_{\mathrm{df}} / M_{\text {predialyzer }}\right)$ contributed more to cytokine removal than adsorption $\left(\% M_{\mathrm{ad}} / M_{\text {predialyzer }}\right)$ for IL-4, TNF$\alpha$, and MCP-1. At $1 \mathrm{~h}$ after CVVHD initiation, total removal ( $\left.\% M_{\text {total }} / M_{\text {predialyzer }}\right)$ ranged from $-12.24 \%$ (IL8 ) to $10.27 \%$ (TNF- $\alpha$ ). The total cytokine removal rates remained stable over the observation period except for VEGF which rose due to increased adsorption. At $48 \mathrm{~h}$, $\% M_{\text {total }} / M_{\text {predialyzer }}$ varied from $-19.06 \%$ (IFN- $\gamma$ ) to 43.54\% (VEGF).

\section{Discussion}

This is the first study which investigated the transport characteristics of 12 molecules across the EMiC2 membrane. The key findings were: first that the plasma concentrations of all molecules declined over $48 \mathrm{~h}$, except for EGF. Second, the effluent clearance rates were low for most cytokines, except for IL-4, IL-8, and MCP-1. Third, 
Table 2 Pre-filter cytokine concentrations at pre-determined time points

\begin{tabular}{|c|c|c|c|c|c|c|}
\hline Cytokines (pg/ml) & TO $(n=12)$ & $\mathrm{T} 1(n=12)$ & T6 $(n=12)$ & $\mathrm{T} 24(n=11)$ & T48 $(n=7)$ & $p$ value* \\
\hline $\mathrm{IL}-2$ & $\begin{array}{l}2.68 \\
(0,4.18)\end{array}$ & $\begin{array}{l}1.21 \\
(0,2.73)\end{array}$ & $\begin{array}{l}0 \\
(0,2.67)\end{array}$ & $\begin{array}{l}0 \\
(0,3.32)\end{array}$ & $\begin{array}{l}0 \\
(0,4.75)\end{array}$ & $<0.001$ \\
\hline $\mathrm{IL}-4$ & $\begin{array}{l}1.30 \\
(1.17,1.92)\end{array}$ & $\begin{array}{l}1.4 \\
(1.16,2.03)\end{array}$ & $\begin{array}{l}1.36 \\
(1.15,1.84)\end{array}$ & $\begin{array}{l}1.19 \\
(0.99,2.24)\end{array}$ & $\begin{array}{l}1.19 \\
(0.99,1.94)\end{array}$ & $<0.001$ \\
\hline$\| L-6$ & $\begin{array}{l}311.03 \\
(30.92,1175.13)\end{array}$ & $\begin{array}{l}84.22 \\
(35.02,1336.48)\end{array}$ & $\begin{array}{l}80.64 \\
(30.12,1273.17)\end{array}$ & $\begin{array}{l}27.48 \\
(23.33,548.69)\end{array}$ & $\begin{array}{l}90.82 \\
(14.4,153.54)\end{array}$ & $<0.001$ \\
\hline$\| L-8$ & $\begin{array}{l}83.21 \\
(55.10,588.14)\end{array}$ & $\begin{array}{l}74.15 \\
(21.79,446.88)\end{array}$ & $\begin{array}{l}88.83 \\
(20.82,356.09)\end{array}$ & $\begin{array}{l}60.44 \\
(22.65,222.59)\end{array}$ & $\begin{array}{l}37.28 \\
(19.99,77.22)\end{array}$ & $<0.001$ \\
\hline $\mathrm{IL}-10$ & $\begin{array}{l}9.35 \\
(4.38,25.46)\end{array}$ & $\begin{array}{l}10.04 \\
(4.35,27.55)\end{array}$ & $\begin{array}{l}9.03 \\
(3.96,19.89)\end{array}$ & $\begin{array}{l}6.58 \\
(3.32,9.42)\end{array}$ & $\begin{array}{l}4.95 \\
(1.54,6.93)\end{array}$ & 0.008 \\
\hline VEGF & $\begin{array}{l}41.25 \\
(21.78,64.97)\end{array}$ & $\begin{array}{l}35.86 \\
(10.46,59.43)\end{array}$ & $\begin{array}{l}29.00 \\
(14.9,35.68)\end{array}$ & $\begin{array}{l}30.28 \\
(20.77,39.77)\end{array}$ & $\begin{array}{l}28.64 \\
(13.93,38.23)\end{array}$ & $<0.001$ \\
\hline IFN- $\gamma$ & $\begin{array}{l}1.59 \\
(1.31,1.92)\end{array}$ & $\begin{array}{l}1.62 \\
(0,2.76)\end{array}$ & $\begin{array}{l}1.01 \\
(0,2.34)\end{array}$ & $\begin{array}{l}0 \\
(0,1.89)\end{array}$ & $\begin{array}{l}0 \\
(0,1.46)\end{array}$ & $<0.001$ \\
\hline TNF-a & $\begin{array}{l}5.84 \\
(4.55,18.03)\end{array}$ & $\begin{array}{l}5.30 \\
(3.2,13.33)\end{array}$ & $\begin{array}{l}4.63 \\
(3.44,13.83)\end{array}$ & $\begin{array}{l}4.14 \\
(2.85,9.1)\end{array}$ & $\begin{array}{l}4.08 \\
(3.14,4.61)\end{array}$ & $<0.001$ \\
\hline $\mathrm{IL}-1 \mathrm{a}$ & $\begin{array}{l}0.72 \\
(0,1.59)\end{array}$ & $\begin{array}{l}0.24 \\
(0,1.27)\end{array}$ & $\begin{array}{l}0 \\
(0,1.07)\end{array}$ & $\begin{array}{l}0 \\
(0,1.29)\end{array}$ & $\begin{array}{l}0.56 \\
(0,1.33)\end{array}$ & $<0.001$ \\
\hline$\| L-1 \beta$ & $\begin{array}{l}1.37 \\
(0.5,2.79)\end{array}$ & $\begin{array}{l}1.09 \\
(0,1.71)\end{array}$ & $\begin{array}{l}1.19 \\
(0,1.77)\end{array}$ & $\begin{array}{l}0 \\
(0,1.47)\end{array}$ & $\begin{array}{l}0 \\
(0,1.64)\end{array}$ & $<0.001$ \\
\hline MCP-1 & $\begin{array}{l}571.9 \\
(335.2,1436.69)\end{array}$ & $\begin{array}{l}516.75 \\
(311.89,779.07)\end{array}$ & $\begin{array}{l}457.74 \\
(275.88,646.24)\end{array}$ & $\begin{array}{l}316.63 \\
(201.93,742.44)\end{array}$ & $\begin{array}{l}262.63 \\
(116.93,451.12)\end{array}$ & $<0.001$ \\
\hline EGF & $\begin{array}{l}1.34 \\
(0,1.49)\end{array}$ & $\begin{array}{l}0 \\
(0,1.66)\end{array}$ & $\begin{array}{l}1.30 \\
(0,1.62)\end{array}$ & $\begin{array}{l}1.38 \\
(0,1.68)\end{array}$ & $\begin{array}{l}1.76 \\
(0,1.84)\end{array}$ & $<0.001$ \\
\hline
\end{tabular}

Values expressed as median (interquartile range)

Normal range from literature for baseline cytokine concentrations in sepsis (in pg/mL):

IL-2 = 0 (0-108.5); IL-4=8.88 (93.2-202.7); IL-6=376-2375; TNF- $\alpha=4.43$ to $33 ; \mathrm{IL}-8=215$ to $1349 ; \mathrm{IL}-1 \beta=0$ to $66.02 ; \mathrm{IL}-10=88.55$ to $638 ; \mathrm{IFN}-\gamma=20.82$ to $275 \mathrm{pg} / \mathrm{mL}$; $\mathrm{MCP}-1=454.2(97.4-22,000) ; \mathrm{EGF}=35.9 \pm 58.03, \mathrm{VEGF}=27.58$ to $1082, \mathrm{IL}-1 \mathrm{a}=0.12$ to $0.36 \mathrm{pg} / \mathrm{mL}[25-34]$

IL interleukin, VEGF vascular endothelial growth factor, IFN interferon, TNF tumour necrosis factor, MCP monocyte chemoattractant protein, EGF epidermal growth factor

* $p$ value for differences in cytokine concentrations for all time points using generalized estimating equations (GEE)

minimal or negative adsorption was observed for all cytokines. Finally, the total removal rates and contributions from CVVHD and adsorption were heterogeneous but were mostly low to moderate.

Extracorporeal blood purification to attenuate the effects of pro-inflammatory and anti-inflammatory mediators in sepsis remains a controversial issue. Although some studies showed potentially promising results and a reduction in cytokine concentrations in plasma with particular extracorporeal techniques, it is not always clear whether these declines in cytokine levels are related to the filter, or simply a reflection of the dynamic nature of sepsis [10]. Baseline cytokine concentrations of our patient cohort were similar or lower than previous reports in the literature [32-41]. This possibly reflects the heterogeneity in phenotypes and severity of sepsis and also underlines the complexity of cytokine profile interpretation in this setting. Whilst some cytokines are proinflammatory and associated with poor outcomes [42], others have a potentially beneficial role. For instance, EGF represents tissue recovery or regeneration after injury and is associated with cellular proliferation and survival in sepsis [43].

Previous studies explored cytokine changes and cytokine removal by the $\mathrm{EMiC} 2$ filter, but none have fully investigated the various mechanisms of cytokine clearance in critically ill patients with sepsis [25-28]. Despite reported decent removal of IL-6, IL-8, IL-1 $\beta$, and TNF- $\alpha$ (MW 8.4-25 kDa) in-vitro, the effluent clearance rates varied markedly in humans [26, 27, 29]. Several factors might affect in vivo clearance, e.g. duration of blood contact, binding to protein or plasma components, dialysate rate or ultrafiltration rate, molecular weight, serum concentration, or sampling time after filter installation. In a previous study using a $\mathrm{HCO}$ membrane, a decline in plasma IL-1ra and IL-6 was observed in patients with high baseline concentrations [11]. This corresponds with our results showing that serum IL-2, IL-6, and IL- $1 \beta$ concentrations were positively correlated with effluent clearance rates. We also noted that the effluent clearance rates remained constant over time which may be explained by the use of citrate-based anticoagulation [44]. Citrate 


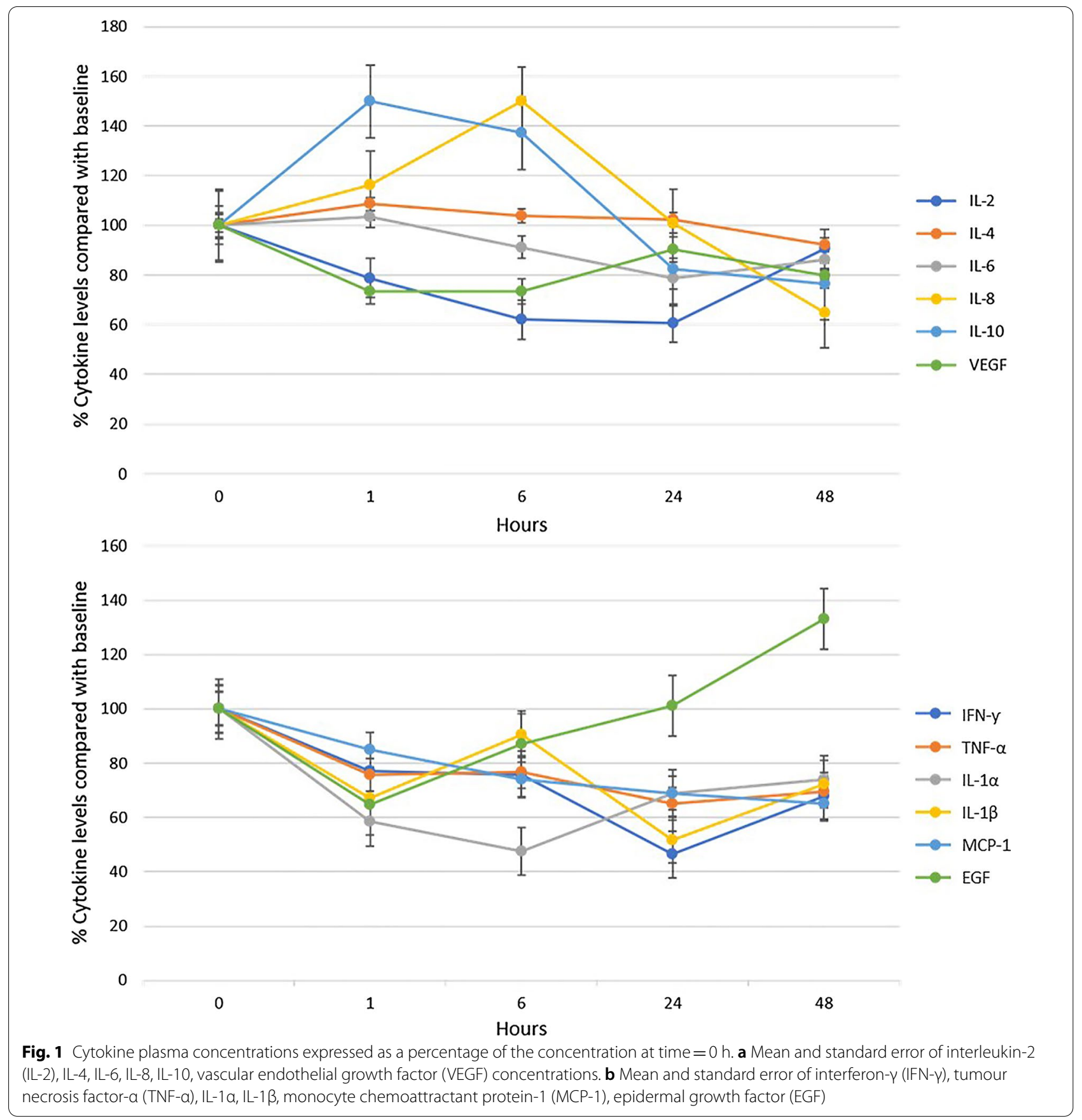

can prolong filter life by reducing both filter clotting (i.e. thrombosis within the lumen of the filter) and clogging (i.e. formation of protein layer which progressively obliterates the membrane pores) $[45,46]$.

The contribution of adsorption to total cytokine removal with EMiC2 filters was minimal. We noted a small degree of adsorption of IL-2 and VEGF which contributed to total mass removal. However, "negative adsorption" was also observed for all cytokines consistent with similar reports in the literature $[15,28,31]$. The mechanisms for this "de-sorption" phenomenon are unclear and might be explained by effects of haemoconcentration on the outflow side from fluid removal albeit in a significantly smaller degree than in CVVH mode, release of previously bound cytokines from cells [47], activation of the inflammatory system through reverse diffusion [28], the 'back-filtration/back-diffusion' phenomenon including the movement of molecules from a 
Table 3 Serial cytokine clearance rates by adsorption and diffusion at 1, 6, 24, and $48 \mathrm{~h}$ after continuous veno-venous haemodialysis initiation and time-weighted mean clearance rates

\begin{tabular}{|c|c|c|c|c|c|}
\hline Cytokines & T1 & T6 & T24 & T48 & Time-weighted mean \\
\hline \multicolumn{6}{|l|}{ IL-2 } \\
\hline Adsorption $(n=6)$ & $\begin{array}{l}3.0 \\
(-21.9,72)\end{array}$ & $\begin{array}{l}18.5 \\
(11.5,20.6)\end{array}$ & $\begin{array}{l}18.8 \\
(-3.6,75)\end{array}$ & $\begin{array}{l}6.4 \\
(-4.0,9.8)\end{array}$ & $\begin{array}{l}9.8 \\
(-3.8,46.3)\end{array}$ \\
\hline Effluent $(n=6)$ & $\begin{array}{l}0 \\
(0,24.2)\end{array}$ & $\begin{array}{l}0 \\
(0,0)\end{array}$ & $\begin{array}{l}0 \\
(0,0)\end{array}$ & $\begin{array}{l}0 \\
(0,0)\end{array}$ & $\begin{array}{l}0 \\
(0,0)\end{array}$ \\
\hline \multicolumn{6}{|l|}{$\mid \mathrm{L}-4$} \\
\hline Adsorption ( $n=12$ ) & $\begin{array}{l}-54.2 \\
(-107.7,-38.7)\end{array}$ & $\begin{array}{l}-61.0 \\
(-75.3,-44.2)\end{array}$ & $\begin{array}{l}-78.1 \\
(-91.7,-46.9)\end{array}$ & $\begin{array}{l}-61.3 \\
(-67.8,-50.8)\end{array}$ & $\begin{array}{l}-64.0 \\
(-91.2,-43.4)\end{array}$ \\
\hline Effluent $(n=12)$ & $\begin{array}{l}55.2 \\
(38.9,69.0)\end{array}$ & $\begin{array}{l}55.9 \\
(50,66.0)\end{array}$ & $\begin{array}{l}59.3 \\
(52.1,72.2)\end{array}$ & $\begin{array}{l}68.9 \\
(43.2,83.9)\end{array}$ & $\begin{array}{l}55.4 \\
(51.3,69.9)\end{array}$ \\
\hline \multicolumn{6}{|l|}{ IL-6 } \\
\hline Adsorption $(n=12)$ & $\begin{array}{l}-15.3 \\
(-21.6,-3.1)\end{array}$ & $\begin{array}{l}-4.9 \\
(-11.5,4.8)\end{array}$ & $\begin{array}{l}-13.3 \\
(-20.8,-1.3)\end{array}$ & $\begin{array}{l}-10.6 \\
(-13.1,-5.7)\end{array}$ & $\begin{array}{l}-11.5 \\
(-18.1,0.9)\end{array}$ \\
\hline Effluent $(n=12)$ & $\begin{array}{l}10.2 \\
(6.1,17.3)\end{array}$ & $\begin{array}{l}9.0 \\
(8.1,10.6)\end{array}$ & $\begin{array}{l}5.6 \\
(2.4,20.5)\end{array}$ & $\begin{array}{l}2.2 \\
(0,6.8)\end{array}$ & $\begin{array}{l}7.8 \\
(4.1,15.7)\end{array}$ \\
\hline \multicolumn{6}{|l|}{ |L-8 } \\
\hline Adsorption $(n=12)$ & $\begin{array}{l}-44.5 \\
(-66,-23)\end{array}$ & $\begin{array}{l}-42.3 \\
(-224.1,-19)\end{array}$ & $\begin{array}{l}-50 \\
(-243.3,-28.8)\end{array}$ & $\begin{array}{l}-61.8 \\
(-100.9,-2.9)\end{array}$ & $\begin{array}{l}-48.9 \\
(-144.7,-19.0)\end{array}$ \\
\hline Effluent $(n=12)$ & $\begin{array}{l}37.7 \\
(26.4,47.8)\end{array}$ & $\begin{array}{l}49.0 \\
(40.8,87.5)\end{array}$ & $\begin{array}{l}62.7 \\
(24.2,116.3)\end{array}$ & $\begin{array}{l}32.7 \\
(16.2,103.1)\end{array}$ & $\begin{array}{l}63.8 \\
(34.7,93.6)\end{array}$ \\
\hline \multicolumn{6}{|l|}{ IL-10 } \\
\hline Adsorption $(n=12)$ & $\begin{array}{l}-5.9 \\
(-16.7,1.6)\end{array}$ & $\begin{array}{l}3.5 \\
(-10.0,7.1)\end{array}$ & $\begin{array}{l}-6.6 \\
(-10.1,8.2)\end{array}$ & $\begin{array}{l}-17.2 \\
(-25.7,-8.5)\end{array}$ & $\begin{array}{l}-7.1 \\
(-10.7,16.9)\end{array}$ \\
\hline Effluent $(n=12)$ & $\begin{array}{l}0.5 \\
(0,10.0)\end{array}$ & $\begin{array}{l}0 \\
(0,1.8)\end{array}$ & $\begin{array}{l}0 \\
(0,1.8)\end{array}$ & $\begin{array}{l}9.6 \\
(4.5,21.3)\end{array}$ & $\begin{array}{l}2.2 \\
(0.3,5.5)\end{array}$ \\
\hline \multicolumn{6}{|l|}{ VEGF } \\
\hline Adsorption $(n=9)$ & $\begin{array}{l}-2.7 \\
(-22.8,11.2)\end{array}$ & $\begin{array}{l}-2.6 \\
(-6.9,10.6)\end{array}$ & $\begin{array}{l}4.0 \\
(-7.4,17.2)\end{array}$ & $\begin{array}{l}37.7 \\
(0.5,100)\end{array}$ & $\begin{array}{l}4.0 \\
(-7.4,16.9)\end{array}$ \\
\hline Effluent $(n=10)$ & $\begin{array}{l}5.0 \\
(0,17.6)\end{array}$ & $\begin{array}{l}0 \\
(0,20.2)\end{array}$ & $\begin{array}{l}0 \\
(0,0)\end{array}$ & $\begin{array}{l}0 \\
(0,16.8)\end{array}$ & $\begin{array}{l}3.4 \\
(0,19.2)\end{array}$ \\
\hline \multicolumn{6}{|l|}{ IFN- $\gamma$} \\
\hline Adsorption $(n=7)$ & $\begin{array}{l}0 \\
(-20.0,24.1)\end{array}$ & $\begin{array}{l}7.9 \\
(4.4,12.3)\end{array}$ & $\begin{array}{l}16.6 \\
(-20.4,5.7)\end{array}$ & $\begin{array}{l}-16.1 \\
(-20.9,-4.5)\end{array}$ & $\begin{array}{l}-2.3 \\
(-20.3,11.2)\end{array}$ \\
\hline Effluent $(n=7)$ & $\begin{array}{l}0 \\
(0,0)\end{array}$ & $\begin{array}{l}0 \\
(0,0)\end{array}$ & $\begin{array}{l}0 \\
(0,0)\end{array}$ & $\begin{array}{l}0 \\
(0,0)\end{array}$ & $\begin{array}{l}0 \\
(0,0)\end{array}$ \\
\hline \multicolumn{6}{|l|}{ TNF-a } \\
\hline Adsorption $(n=12)$ & $\begin{array}{l}-5.3 \\
(-10.6,3.5)\end{array}$ & $\begin{array}{l}-7.0 \\
(-12.3,4.1)\end{array}$ & $\begin{array}{l}-22.8 \\
(-38.8,-13.4)\end{array}$ & $\begin{array}{l}-19.3 \\
(-23.4,-0.6)\end{array}$ & $\begin{array}{l}-10.6 \\
(-23.3,4.1)\end{array}$ \\
\hline Effluent $(n=12)$ & $\begin{array}{l}11.6 \\
(0,17.0)\end{array}$ & $\begin{array}{l}19.0 \\
(13.9,30.7)\end{array}$ & $\begin{array}{l}26.5 \\
(13.5,33.2)\end{array}$ & $\begin{array}{l}22.7 \\
(18.5,28.8)\end{array}$ & $\begin{array}{l}19.0 \\
(9.0,28.4)\end{array}$ \\
\hline \multicolumn{6}{|l|}{ IL-1a } \\
\hline Adsorption $(n=6)$ & $\begin{array}{l}-7.2 \\
(-13.1,69)\end{array}$ & $\begin{array}{l}-5.6 \\
(-30.2,5.0)\end{array}$ & $\begin{array}{l}-26.1 \\
(-38.9,-7.7)\end{array}$ & $\begin{array}{l}-11.4 \\
(-46.1,48.1)\end{array}$ & $\begin{array}{l}-10.2 \\
(-23.2,4.1)\end{array}$ \\
\hline Effluent $(n=6)$ & $\begin{array}{l}0 \\
(0,0)\end{array}$ & $\begin{array}{l}0 \\
(0,35.0)\end{array}$ & $\begin{array}{l}0 \\
(0,15.2)\end{array}$ & $\begin{array}{l}0 \\
(0,9.3)\end{array}$ & $\begin{array}{l}0 \\
(0,8.6)\end{array}$ \\
\hline \multicolumn{6}{|l|}{$\mid L-1 \beta$} \\
\hline Adsorption $(n=7)$ & $\begin{array}{l}-12.2 \\
(-62.3,0)\end{array}$ & $\begin{array}{l}22.5 \\
(-3.6,56)\end{array}$ & $\begin{array}{l}-21.1 \\
(-46.0,-10.2)\end{array}$ & $-9.2^{\mathrm{a}}$ & $-8.6(-21.1,3.1)$ \\
\hline Effluent $(n=7)$ & $\begin{array}{l}0 \\
(0,29.0)\end{array}$ & $\begin{array}{l}0 \\
(0,20.0)\end{array}$ & $\begin{array}{l}30.0 \\
(0,61.4)\end{array}$ & $0^{\mathrm{a}}$ & $\begin{array}{l}0 \\
(0,31.8)\end{array}$ \\
\hline \multicolumn{6}{|l|}{ MCP-1 } \\
\hline Adsorption $(n=12)$ & $\begin{array}{l}-31.8 \\
(-19.9,-13.4)\end{array}$ & $\begin{array}{l}-23.1 \\
(-25.4,-17.8)\end{array}$ & $\begin{array}{l}-30.5 \\
(-35.7,-10.9)\end{array}$ & $\begin{array}{l}-22.9 \\
(-40.5,-4.8)\end{array}$ & $\begin{array}{l}-24.9 \\
(-35.7,-10.9)\end{array}$ \\
\hline
\end{tabular}


Table 3 (continued)

\begin{tabular}{|c|c|c|c|c|c|}
\hline Cytokines & $\mathrm{T} 1$ & T6 & T24 & T48 & Time-weighted mean \\
\hline Effluent $(n=12)$ & $\begin{array}{l}33.5 \\
(29.7,46.6)\end{array}$ & $\begin{array}{l}38.2 \\
(35.0,43.0)\end{array}$ & $\begin{array}{l}31.3 \\
(18.0,50)\end{array}$ & $\begin{array}{l}32.9 \\
(24.5,46.6)\end{array}$ & $\begin{array}{l}33.9 \\
(21.6,43.6)\end{array}$ \\
\hline \multicolumn{6}{|l|}{ EGF } \\
\hline Adsorption $(n=5)$ & $\begin{array}{l}0.9 \\
(0.7,1.7)\end{array}$ & $\begin{array}{l}8.2 \\
(0,15.1)\end{array}$ & $\begin{array}{l}-7.3 \\
(-14.8,-4.8)\end{array}$ & $\begin{array}{l}-0.2 \\
(-43.0,3.7)\end{array}$ & $\begin{array}{l}0 \\
(-12,3.4)\end{array}$ \\
\hline Effluent $(n=7)$ & $\begin{array}{l}0 \\
(0,0)\end{array}$ & $\begin{array}{l}0 \\
(0,0)\end{array}$ & $\begin{array}{l}0 \\
(0,0)\end{array}$ & $\begin{array}{l}0 \\
(0,0)\end{array}$ & $\begin{array}{l}0 \\
(0,0)\end{array}$ \\
\hline
\end{tabular}

Values expressed as median (interquartile range)

IL interleukin, VEGF vascular endothelial growth factor, IFN interferon, TNF tumour necrosis factor, MCP monocyte chemoattractant protein, EGF epidermal growth factor, $\mathrm{T} 1=1 \mathrm{~h}, \mathrm{~T} 6=6 \mathrm{~h}, \mathrm{~T} 24=24 \mathrm{~h}$, and $\mathrm{T} 48=48 \mathrm{~h}$

a Only one observation

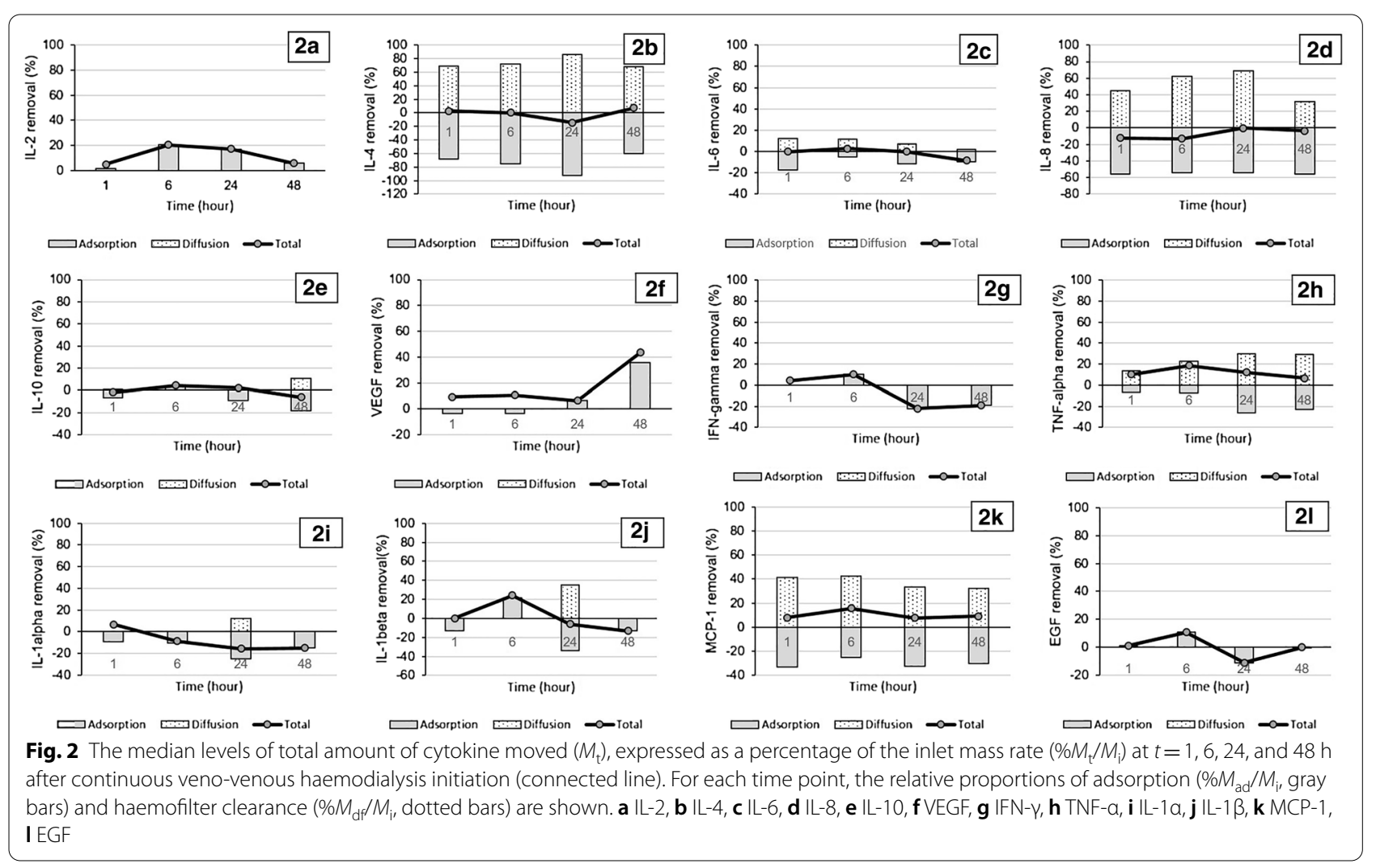

higher concentration in the dialysate to a lower concentration in the blood at the distal end of the hollow fibres in high-flux membranes [48], cytokine induction from dialyzer bio-incompatibility [49], activation or deactivation by enzymes after sampling, or sampling errors.

We found that the changes in cytokine concentrations seen in plasma were discordant with the extent of removal by clearance and adsorption. Despite a decline in serum concentrations, we found low total mass removal rates across the filter for most cytokines. The clearance rates were highest for IL-4, IL-8, and MCP-1, but their plasma reduction ratios varied significantly from -13.46 to $-59.94 \%$. Although VEGF showed the highest total mass removal at $48 \mathrm{~h}$, the reduction ratio was $-34.88 \%$ which was lower than others.

Some of our findings are compatible with data in the literature but not all. For instance, a previous study demonstrated higher IL- 6 and IL-8 clearance by the EMiC2 filter than the standard membrane, but showed no significant impact on plasma cytokine concentrations [26]. Another 
study showed comparable IL-6 clearance between the EMiC2 and high-flux membrane [27]. Similarly, studies using the EMiC2 or $\mathrm{HCO}$ membranes reported no changes in plasma concentrations despite detectable clearance in the ultrafiltrate $[11,15,28,50]$. These results in the literature, together with our findings highlight that a rise or fall in serum concentrations during KRT might be related to factors other than extracorporeal removal, e.g. changes in cytokine production, endogenous clearance, intradialytic cytokine release, general improvement of the underlying disease, or response to treatment [51]. It should also be noted that cytokine half-lives are extremely short. (Additional file 6) Thus, their endogenous metabolism might be more rapid than clearance during extracorporeal therapy. In general, the kidneys contribute to $15-20 \%$ of cytokine metabolism [52]. Our study showed that CVVHD using the EMiC2 filter contributed little to total clearance of most cytokines. This suggests that extracorporeal removal does not substitute renal clearance, similar to lactate removal [53]. In addition, the interaction of soluble forms and their endogenous modulators (receptors or antagonists) indicates different states of immune activation. Thus, the measurement of plasma cytokine concentrations in isolation might not reflect the true immune status or indicate the impact of extracorporeal removal on the dynamics of that particular cytokine system [54].

This is the first study to investigate a comprehensive panel of molecules which are representative of pro- and anti-inflammatory cytokines in real clinical settings. Adsorption and diffusive clearances were evaluated extensively by determination of their clearance rates, and mass balances across the membrane over a 48 -h period. However, some limitations need to be acknowledged. First, the objective of this pilot study was to investigate the mechanistic impact of using the $\mathrm{EMiC} 2$ filter. It was not powered to assess an association with clinical outcomes. Second, we did not intend to compare the EMiC2 filter with other filters. Therefore, there was no control group. However, this is an exploratory study to characterize the transport characteristics of cytokines when using the $\mathrm{EMiC} 2$ filter. This investigation is essential before proceeding to larger clinical studies investigating the role of blood purification with the $\mathrm{EMiC} 2$ filter as an adjunctive therapy in sepsis. Third, we selected 12 different molecules but did not measure all potential pro- and anti-inflammatory cytokines. We acknowledge that our conclusions only apply to the cytokines measured and that it is possible that other cytokines or medium-sized molecules are removed at higher quantities when using the EMiC2 filter. Fourth, the cohort of included patients was heterogenous and there were some patients with undetectable cytokine concentrations throughout the whole study period. Given the mechanistic nature of this project, we only included subjects with detectable predialyzer concentrations when analysing the effluent and adsorptive clearance rates and mass balances. Although there was minimal correlation between initial cytokine concentrations and clearance rates, we are unable to comment on whether cytokine removal in patients with different cytokine concentrations, including cytokine storm/septic shock would have different clinical effects. We acknowledge that cytokines may have different effects independent of the concentration, including paracrine and endocrine actions [55]. Finally, our aim was to describe clearance and adsorption of cytokines during CVVHD with an EMiC2 filter. Although we showed that only small amounts of cytokines were actually removed, we acknowledge that we cannot exclude any immunomodulatory effects $[56,57]$.

\section{Conclusion}

Our study has shown that in patients with sepsis and acute kidney injury requiring KRT with the $\mathrm{EMiC} 2$ filter, clearance of cytokines by CVVHD was modest and adsorption was minor. We observed a decline in serum concentrations of most cytokines during the study period but were unable to detect an obvious correlation between serum concentration and cytokine clearance. The results suggest that mechanisms other than extracorporeal removal contribute to changes in plasma cytokine concentrations. Further work to determine the role of the $\mathrm{EMiC2}$ filter in clinical practice is required.

\section{Supplementary Information}

The online version contains supplementary material available at https://doi. org/10.1186/s13054-021-03476-X.

Additional file 1. Sites of sampling from the CVVHD circuit.

Additional file 2. Reduction ratio of cytokine concentrations at $t=1$ ( $n=$ $12), 6(n=12), 24(n=11)$, and $48(n=7)$ hours compared with baseline pre-filter concentrations (\%)

Additional file 3. Clearance rates ( $\mathrm{mL} / \mathrm{min}$ ) of cytokines by adsorption (pink bars) and effluent (blue bars) over time visualized as box and whisker plots (horizontal bars indicate median values).

Additional file 4. Spearman's correlation between serum levels and clearances by adsorption and effluent.

Additional file 5. Mass balances for all cytokines (pg/min): Mass removal rate by adsorption (Mad), mass removal rate by dialysis (Mdf), and total mass removal rate $(\mathrm{Mt})$.

Additional file 6. Cytokine half-lives as reported in literature.

\section{Abbreviations}

AKI: Acute kidney injury; APACHE: Acute Physiologic and Chronic Health Evaluation; BPT: Blood purification therapy; Ceff: Effluent concentration; Cleff: Effluent clearance; Clmean: Time weighted average clearance; Cltotal: Total clearance; Cpredialyzer: Predialyzer serum concentration; Cpostdialyzer: Postdialyzer serum concentration; CKRT: Continuous kidney replacement therapy; CWHD: Continuous veno-venous haemodialysis; CVHDF: Continuous 
veno-venous haemodiafiltration; EGF: Epidermal growth factor; HCO: High cut-off; Hct: Haematocrit; HVHF: High-volume haemofiltration; IFN: Interferon; ICU: Intensive care unit; IL: Interleukin; IQR: Interquartile range; $\mathrm{K}_{\mathrm{ad}}$ : Adsorptive clearance; $k D a$ : Kilodalton; KRT: Kidney replacement therapy; $M_{\mathrm{ad}}$ : Mass removal by adsorption; $M_{d f}$ : Mass removal by dialysis; $M_{\text {predialyzer: }}$ Predialyzer mass balance; $M_{\text {postdialyzer: }}$ Postdialyzer mass balance; $M_{t}$ : Total mass removal rate; MCO: Medium cut-off; MCP: Monocyte chemoattractant protein; PMMA Polymethyl methacrylate; PS: Polysulfone; $\mathrm{Q}_{\mathrm{b}}$ : Blood flow rate; $\mathrm{Q}_{\mathrm{i}}$ : Predialyzer plasma flow rate; $Q_{d}$ : Dialysate flow rate; $Q_{0}$ : Postdialyzer plasma flow rate; $Q_{u f}$ : Ultrafiltration rate; RR: Reduction ratio; SOFA: Sequential Organ Failure Assessment; sTNF-Rs: Soluble tumour necrosis factor-receptors; TNF: Tumour necrosis factor; VEGF: Vascular endothelial growth factor.

\section{Acknowledgements}

We would like to acknowledge the support from the research nurse, Katie Lei, and renal nurses, Helen Dickie and Linda Tovey, in the critical care department at Guy's \& St Thomas' Hospital who helped recruiting patients and taking the samples according to the study protocol.

\section{Authors' contributions}

The protocol was developed by MO, who also oversaw the project. AH recruited patients, collected the samples and reviewed the manuscript. NL performed the statistical analysis, wrote the first draft and handled subsequent versions of the manuscript. SC contributed to the statistical analysis and reviewed the manuscript. LC helped developing the protocol and reviewed the manuscript. All authors approved the final manuscript.

\section{Funding}

The study was partially supported by an independent research grant from Fresenius Medical. The company was not involved in the design of the protocol, conduct of the study, interpretation of the results and writing of the manuscript.

\section{Availability of data and materials}

The datasets used and/or analysed during the current study are available from the corresponding author on reasonable request.

\section{Ethical approval and consent to participate}

The study was approved by the National Research Ethics Committee (16/ LO/0313). Written informed consent was obtained from all patients or their legal representatives.

\section{Consent for publication}

Patients and/or legal representatives gave written consent to participating in the study and for their anonymous data to be included in publications.

\section{Competing interests}

Dr. Ostermann has received speaker honoraria and research funding from Fresenius Medical. All other authors declare no conflicts of interests.

\section{Author details}

${ }^{1}$ Department of Critical Care, Guy's and St Thomas' Hospital, King's College London, NHS Foundation Trust, 249 Westminster Bridge Road, London SE1 7EH, UK. ${ }^{2}$ Division of Nephrology and Excellence Centre for Critical Care Nephrology, King Chulalongkorn Memorial Hospital, Bangkok, Thailand. ${ }^{3}$ Critical Care Nephrology Research Unit, Chulalongkorn University, Bangkok, Thailand. ${ }^{4}$ Zorgsaam Terneuzen, Rotterdam, The Netherlands. ${ }^{5}$ Medical Research Council Clinical Trials Unit, University College London, London, UK.

Received: 16 November 2020 Accepted: 20 January 2021

Published online: 28 January 2021

\section{References}

1. Singer M, Deutschman CS, Seymour CW, Shankar-Hari M, Annane D, Bauer $\mathrm{M}$, et al. The third international consensus definitions for sepsis and septic shock (Sepsis-3). JAMA. 2016;315(8):801-10.

2. Rhodes A, Evans LE, Alhazzani W, Levy MM, Antonelli M, Ferrer R, et al. Surviving sepsis campaign: international guidelines for management of sepsis and septic shock: 2016. Crit Care Med. 2017;45(3):486-552.
3. Peerapornratana S, Manrique-Caballero CL, Gómez H, Kellum JA. Acute kidney injury from sepsis: current concepts, epidemiology, pathophysiology, prevention and treatment. Kidney Int. 2019;96(5):1083-99.

4. Ronco C, Tetta C, Mariano F, Wratten ML, Bonello M, Bordoni V, et al. Interpreting the mechanisms of continuous renal replacement therapy in sepsis: the peak concentration hypothesis. Artif Organs. 2003;27(9):792-801.

5. Ronco C. Endotoxin removal: history of a mission. Blood Purif. 2014;37(Suppl 1):5-8.

6. Peng ZY, Bishop JV, Wen XY, Elder MM, Zhou F, Chuasuwan A, et al. Modulation of chemokine gradients by apheresis redirects leukocyte trafficking to different compartments during sepsis, studies in a rat model. Crit Care. 2014;18(4):R141.

7. Honoré PM, Matson JR. Extracorporeal removal for sepsis: acting at the tissue level-the beginning of a new era for this treatment modality in septic shock. Crit Care Med. 2004;32(3):896-7.

8. Rimmelé T, Kaynar AM, McLaughlin JN, Bishop JV, Fedorchak MV, Chuasuwan A, et al. Leukocyte capture and modulation of cell-mediated immunity during human sepsis: an ex vivo study. Crit Care. 2013;17(2):R59.

9. Srisawat N, Tungsanga S, Lumlertgul N, Komaenthammasophon C, Peerapornratana S, Thamrongsat $\mathrm{N}$, et al. The effect of polymyxin B hemoperfusion on modulation of human leukocyte antigen DR in severe sepsis patients. Crit Care. 2018;22(1):279.

10. Atan R, Crosbie D, Bellomo R. Techniques of extracorporeal cytokine removal: a systematic review of the literature. Blood Purif. 2012;33(1-3):88-100.

11. Morgera S, Slowinski T, Melzer C, Sobottke V, Vargas-Hein O, Volk T, et al. Renal replacement therapy with high-cutoff hemofilters: impact of convection and diffusion on cytokine clearances and protein status. Am J Kidney Dis. 2004;43(3):444-53.

12. Haase M, Bellomo R, Baldwin I, Haase-Fielitz A, Fealy N, Davenport P, et al. Hemodialysis membrane with a high-molecular-weight cutoff and cytokine levels in sepsis complicated by acute renal failure: a phase 1 randomized trial. Am J Kidney Dis. 2007;50(2):296-304.

13. Chelazzi C, Villa G, D'Alfonso MG, Mancinelli P, Consales G, Berardi M, et al. Hemodialysis with high cut-off hemodialyzers in patients with multi-drug resistant gram-negative sepsis and acute kidney injury: a retrospective. Case-Control Study Blood Purif. 2016;42(3):186-93.

14. Morgera S, Haase M, Kuss T, Vargas-Hein O, Zuckermann-Becker H, Melzer $C$, et al. Pilot study on the effects of high cutoff hemofiltration on the need for norepinephrine in septic patients with acute renal failure. Crit Care Med. 2006;34(8):2099-104.

15. Atan R, Peck L, Visvanathan K, Skinner N, Eastwood G, Bellomo R, et al. High cut-off hemofiltration versus standard hemofiltration: effect on plasma cytokines. Int J Artif Organs. 2016;39(9):479-86.

16. Villa G, Chelazzi C, Morettini E, Zamidei L, Valente S, Caldini AL, et al. Organ dysfunction during continuous veno-venous high cut-off hemodialysis in patients with septic acute kidney injury: a prospective observational study. PLoS ONE. 2017;12(2):e0172039.

17. Atan R, Peck L, Prowle J, Licari E, Eastwood GM, Storr M, et al. A doubleblind randomized controlled trial of high cutoff versus standard hemofiltration in critically ill patients with acute kidney injury. Crit Care Med. 2018;46(10):e988-94.

18. Boschetti-de-Fierro A, Voigt M, Storr M, Krause B. Extended characterization of a new class of membranes for blood purification: the high cut-off membranes. Int J Artif Organs. 2013;36(7):455-63.

19. Morgera S, Rocktäschel J, Haase M, Lehmann C, von Heymann C, Ziemer $S$, et al. Intermittent high permeability hemofiltration in septic patients with acute renal failure. Intensive Care Med. 2003;29(11):1989-95.

20. Jayaballa M, Bose B, Gangadharan Komala M, Fischer ER, Taper J, Sud K. Effective removal of $\mathrm{K}$-free light chains with hemodialysis using Fresenius Ultraflux ${ }^{\circledR} \mathrm{EMiC}^{\circledR} 2$ dialyser in a patient with myeloma cast nephropathy, with associated cost savings. Blood Purif. 2016;42(2):158-9.

21. Dilken O, Ince C, van der Hoven B, Thijsse S, Ormskerk P, de Geus HRH. Successful reduction of creatine kinase and myoglobin levels in severe rhabdomyolysis using extracorporeal blood purification (CytoSorb ${ }^{\circledR}$ ). Blood Purif. 2020:49(6):743-7.

22. Díaz-Tejeiro R, Regidor D, Morales J, Padrón M, Cueto L, Muñoz MA, et al. Acute renal failure due to rhabdomyolysis. Renal replacement therapy with intermediate cut-off membranes (EMIC2). Nefrologia. 2018;38(6):664-5. 
23. Lyubimova NV, Timofeeva YS, Gromova EG, Kuznetsova LS, Votyakova OM, Kushlinskii NE. Free immunoglobulin light chains as criteria of extracorporeal hemocorrection in patients with monoclonal gammopathies. Bull Exp Biol Med. 2017;163(4):493-6.

24. Schmidt JJ, Hafer C, Clajus C, Hadem J, Beutel G, Schmidt BM, et al. New high-cutoff dialyzer allows improved middle molecule clearance without an increase in albumin loss: a clinical crossover comparison in extended dialysis. Blood Purif. 2012;34(3-4):246-52.

25. Weidhase L, Haussig E, Haussig S, Kaiser T, de Fallois J, Petros S. Middle molecule clearance with high cut-off dialyzer versus high-flux dialyzer using continuous veno-venous hemodialysis with regional citrate anticoagulation: a prospective randomized controlled trial. PLoS ONE. 2019;14(4):e0215823.

26. Eichhorn T, Hartmann J, Harm S, Linsberger I, König F, Valicek G, et al. Clearance of selected plasma cytokines with continuous veno-venous hemodialysis using Ultraflux EMiC2 versus Ultraflux AV1000S. Blood Purif. 2017:44(4):260-6.

27. Weidhase L, de Fallois J, Haußig E, Kaiser T, Mende M, Petros S. Myoglobin clearance with continuous veno-venous hemodialysis using high cutoff dialyzer versus continuous veno-venous hemodiafiltration using high-flux dialyzer: a prospective randomized controlled trial. Crit Care. 2020;24(1):644

28. Balgobin S, Morena M, Brunot V, Besnard N, Daubin D, Platon L, et al. Continuous veno-venous high cut-off hemodialysis compared to continuous veno-venous hemodiafiltration in intensive care unit acute kidney injury patients. Blood Purif. 2018;46(3):248-56.

29. Harm S, Schildböck C, Hartmann J. Cytokine removal in extracorporeal blood purification: an in vitro study. Blood Purif. 2020;49(1-2):33-43.

30. $\mathrm{Ci}-\mathrm{Ca}{ }^{\circledR} \mathrm{CVVHD}$ with Ultraflux ${ }^{\circledR} \mathrm{EMiC}^{\circledR}$ 2. https://www.freseniusmedicalcare .hu/fileadmin/data/masterContent/pdf/Healthcare_Professionals/04_ Acute_Therapies/06_CRRT_and_plasma_filters/Br_CiCa-EMiC2_Produ ct_05_13_GB_w_RGB_.pdf. Accessed 20 Oct 2020.

31. Ricci Z, Ronco C, Bachetoni A, D’Amico G, Rossi S, Alessandri E, et al. Solute removal during continuous renal replacement therapy in critically ill patients: convection versus diffusion. Crit Care. 2006;10(2):R67.

32. Heering P, Morgera S, Schmitz FJ, Schmitz G, Willers R, Schultheiss HP, et al. Cytokine removal and cardiovascular hemodynamics in septic patients with continuous venovenous hemofiltration. Intensive Care Med. 1997;23(3):288-96.

33. Hoffmann JN, Hartl WH, Deppisch R, Faist E, Jochum M, Inthorn D. Hemofiltration in human sepsis: evidence for elimination of immunomodulatory substances. Kidney Int. 1995;48(5):1563-70.

34. Jekarl DW, Kim JY, Ha JH, Lee S, Yoo J, Kim M, et al. Diagnosis and prognosis of sepsis based on use of cytokines, chemokines, and growth factors. Dis Markers. 2019;2019:1089107.

35. Kellum JA, Johnson JP, Kramer D, Palevsky P, Brady JJ, Pinsky MR. Diffusive vs. convective therapy: effects on mediators of inflammation in patient with severe systemic inflammatory response syndrome. Crit Care Med. 1998;26(12):1995-2000.

36. Low C, Syed D, Khan D, Tetik S, Walborn A, Hoppensteadt D, et al. Modulation of interleukins in sepsis-associated clotting disorders: interplay with hemostatic derangement. Clin Appl Thromb Hemost. 2017;23(1):34-9.

37. Lvovschi $V$, Arnaud L, Parizot C, Freund Y, Juillien G, Ghillani-Dalbin P, et al. Cytokine profiles in sepsis have limited relevance for stratifying patients in the emergency department: a prospective observational study. PLoS ONE. 2011;6(12):e28870.

38. Peng Z, Pai P, Hong-Bao L, Rong L, Han-Min W, Chen $\mathrm{H}$. The impacts of continuous veno-venous hemofiltration on plasma cytokines and monocyte human leukocyte antigen-DR expression in septic patients. Cytokine. 2010;50(2):186-91.

39. Pickkers P, Sprong T, Eijk L, Hoeven H, Smits P, Deuren M. Vascular endothelial growth factor is increased during the first 48 hours of human septic shock and correlates with vascular permeability. Shock. 2005;24(6):508-12.

40. van der Flier M, van Leeuwen HJ, van Kessel KP, Kimpen JL, Hoepelman Al, Geelen SP. Plasma vascular endothelial growth factor in severe sepsis. Shock. 2005;23(1):35-8.

41. Kox M, Waalders NJB, Kooistra EJ, Gerretsen J, Pickkers P. Cytokine levels in critically ill patients with COVID-19 and other conditions. JAMA. 2020;324:1565-7

42. Meduri GU, Headley S, Kohler G, Stentz F, Tolley E, Umberger R, et al. Persistent elevation of inflammatory cytokines predicts a poor outcome in ARDS. Plasma IL-1 beta and IL-6 levels are consistent and efficient predictors of outcome over time. Chest. 1995;107(4):1062-73.

43. Klingensmith NJ, Chen CW, Liang Z, Burd EM, Farris AB, Arbiser JL, et al. Honokiol increases CD4+ T cell activation and decreases TNF but fails to improve survival following sepsis. Shock. 2018;50(2):178-86.

44. Fischer KG. Essentials of anticoagulation in hemodialysis. Hemodial Int. 2007;11(2):178-89.

45. Jacobs R, Honoré PM, Bagshaw SM, Diltoer M, Spapen HD. Citrate formulation determines filter lifespan during continuous veno-venous hemofiltration: a prospective cohort study. Blood Purif. 2015;40(3):194-202.

46. Honore PM, Spapen HD. What a clinician should know about a renal replacement membrane? J Transl Int Med. 2018;6(2):62-5.

47. De Vriese AS, Colardyn FA, Philippé JJ, Vanholder RC, De Sutter JH, Lameire $\mathrm{NH}$. Cytokine removal during continuous hemofiltration in septic patients. J Am Soc Nephrol. 1999;10(4):846-53.

48. Santoro A, Guadagni G. Dialysis membrane: from convection to adsorption. NDT Plus. 2010;3(Suppl 1):i36-9.

49. Vaslaki L, Weber C, Mitteregger R, Falkenhagen D. Cytokine induction in patients undergoing regular online hemodiafiltration treatment. Artif Organs. 2000;24(7):514-8.

50. Kade G, Lubas A, Rzeszotarska A, Korsak J, Niemczyk S. Effectiveness of high cut-off hemofilters in the removal of selected cytokines in patients during septic shock accompanied by acute kidney injury-preliminary study. Med Sci Monit. 2016;22:4338-44.

51. Honore PM, Hoste E, Molnár Z, Jacobs R, Joannes-Boyau O, Malbrain M, et al. Cytokine removal in human septic shock: where are we and where are we going? Ann Intensive Care. 2019;9(1):56.

52. Interleukins BV. Clinical pharmacokinetics and practical implications. Clin Pharmacokinet. 1991;21(4):274-84.

53. Levraut J, Ciebiera JP, Jambou P, Ichai C, Labib Y, Grimaud D. Effect of continuous venovenous hemofiltration with dialysis on lactate clearance in critically ill patients. Crit Care Med. 1997;25(1):58-62.

54. Diez-Ruiz A, Tilz GP, Zangerle R, Baier-Bitterlich G, Wachter H, Fuchs D. Soluble receptors for tumour necrosis factor in clinical laboratory diagnosis. Eur J Haematol. 1995;54(1):1-8.

55. Zhang JM, An J. Cytokines, inflammation, and pain. Int Anesthesiol Clin. 2007;45(2):27-37

56. Morgera S, Haase M, Rocktäschel J, Böhler T, von Heymann C, Vargas-Hein $\mathrm{O}$, et al. High permeability haemofiltration improves peripheral blood mononuclear cell proliferation in septic patients with acute renal failure. Nephrol Dial Transplant. 2003;18(12):2570-6.

57. Morgera S, Haase M, Rocktäschel J, Böhler T, Vargas-Hein O, Melzer C, et al. Intermittent high-permeability hemofiltration modulates inflammatory response in septic patients with multiorgan failure. Nephron Clin Pract. 2003;94(3):c75-80.

\section{Publisher's Note}

Springer Nature remains neutral with regard to jurisdictional claims in published maps and institutional affiliations. 